DOI: 10.21892/978-958-5547-66-7.7

\title{
7. Ampliación progresiva de la cobertura vs. principio de sostenibilidad financiera en el Sistema General de Seguridad Social
}

Indy Nazir Lleneris ${ }^{1}$

\section{Introducción}

Con la expedición de la Ley 100 de 1993 —la cual cambio tangencialmente los requisitos para albergar el estatus de pensionadose creó un nuevo sistema de seguridad social integral. La integralidad del sistema devenía de la regulación concentrada de todos los aspectos que comprende la seguridad social (Guerrero, 2008), entre ellos el acceso a la prestación económica denominada pensión. Ahora bien, el sistema integral de seguridad social está constituido por tres aristas: SGSS en Salud, SGSS en Riesgos laborales y el SGSS en Pensiones.

El objeto de estudio de esta investigación se enfoca en este último. Dentro de este sistema existen dos Regímenes de cotización: RAIS — régimen de ahorro individual con solidaridad—, y el RPMPD —régimen de prima media con prestación definida-. El RAIS está dirigido a las cotizaciones individuales, es decir, las realizadas por trabajadores independientes. Mientras que el RPMPD está orientado a los cotizantes vinculados al sector laboral mediante contrato de trabajo. Lo anterior implica, que el trabajador tiene la calidad de empleado, y por tanto, cumple un horario de trabajo, se encuentra subordinado y recibe un salario como retribución directa del servicio prestado.

Dentro del RPMPD existen regímenes especiales, dependiendo a veces de las calidades del trabajador, por ejemplo, cuando el trabajador ejerce funciones de dirección confianza y manejo, y otras veces de las entidades a las cuales pertenece, que pueden ser privadas o públicas.

1 Abogada de la Universidad de Cartagena. Magíster en Derecho de la Universidad de Cartagena. 
En nuestro caso optaremos por el análisis de un régimen especial, a fin de delimitar el campo de estudio ${ }^{2}$. Por ello, elegiremos a un sujeto pasivo, examinando el despliegue normativo que cobija a los empleados públicos.

En este sentido, veremos como el SGSS en pensiones está diseñado con la intención de que el ciudadano pueda pensionarse. Para ello deben cumplirse con dos requisitos: edad y tiempo de servicio.

De otra parte, estudiaremos como la Ley 100 de 1993, dedicó el Capítulo primero del Título segundo a la depuración del sistema, y sostuvo en su artículo décimo que el sistema general de pensiones tiene por objeto garantizar a la población el amparo contra las contingencias derivadas de la vejez, la invalidez y la muerte, mediante el reconocimiento de las pensiones y prestaciones que se determinan en la mencionada ley, así como propender a la ampliación progresiva de cobertura a los segmentos de población no cubiertos con un sistema de pensiones.

No obstante, esta pretensión debe mirarse a la luz de otros principios del orden constitucional que amparan y direccionan el quehacer del sistema. Vale decir que mediante Acto Legislativo 01 de 2005 la Constitución Política de Colombia sufrió una modificación, más exactamente en el artículo 48 de este compendio normativo que consagra el derecho a la seguridad social. El inciso tercero del artículo que nos ocupa, retoma el objeto de la seguridad social, y reza que: "El Estado, con la participación de los particulares, ampliará progresivamente la cobertura...". Del mismo modo, el inciso sexto indica que "el Estado garantizará los derechos, la sostenibilidad financiera del sistema pensional...".

Por tanto, tenemos que el objeto del sistema y el principio de sostenibilidad financiera no responden a intereses similares. Este estudio pretende establecer ciertos tópicos que permiten soportar el enunciado anterior, no obstante, se hace necesario manifestar que este capítulo no agota el asunto en su totalidad, pero a nuestro parecer las reflexiones al respecto

2 Este documento es resultado del proyecto de investigación en curso denominado: "Análisis del SGSS en Pensiones a luz de los Principios Constitucionales que lo orientan: Negación de la Pensión de Vejez a Empleados Públicos", que tiene por objeto Analizar el SGSS en Pensiones, cuando mediante resolución administrativa, se niega la prestación económica a empleados públicos, a la luz de los principios constitucionales que lo orien$\tan$. 
resultan valiosas, a fin de visibilizar cómo los principios orientadores de un sistema podrían estar en contraposición con el sentido inicial del sistema mismo.

\section{El SGSSP en la Constitución Política de 1991}

Sea lo primero señalar, que un fenómeno recurrente dentro del ordenamiento jurídico colombiano, desde la expedición de la Constitución Política de 1991, ha sido la constitucionalización de las diversas ramas del derecho, entre ellas la seguridad social, superando el marco netamente legal —que se había caracterizado por su abulia frente al derecho pensional-y adquiriendo un rango constitucional, que se arguye como soporte, una vez los trabajadores han cumplido con los requisitos para albergar el estatus de pensionados, siendo acreedores del derecho a acceder a una pensión de vejez.

Ahora bien, es preciso recordar que toda norma constitucional es de estructura, y una vez se procedió a la expedición de nuestra carta política, el texto constitucional se orientó bajo la Ideología Demoliberal. La ideología que nos ocupa, se encuentra basada en cuatro pilares o principios fundamentales, como son: el Estado social de derecho, las tendencias socialistas, el liberalismo y la democracia republicana.

Lo anterior, trajo consigo ciertas implicaciones que se vieron reflejadas en la estructura del Estado. Este último pasó de ser un Estado de derecho para convertirse en un Estado social de derecho, el cual pretende establecer un orden económico-social basado en la procura de un mínimo existencial. A este mínimo existencial no solo le atañe el concepto salarial, sino que se vincula con el orden pensional, es decir, con la posibilidad material de que el ciudadano colombiano cuente con un respaldo económico a lo largo de toda su existencia, inclusive cuando se retire de sus labores en ocasión a la edad.

Es por ello que la carta política se dispuso a verificar definitivamente el derecho social del derecho del trabajo, que es el de aminorar las desigualdades e imponer la paz social (Guerrero, 2008). 
En vista de estas orientaciones preliminares de nuestra carta —los principios en que se fundamenta, así como la ideología que pregona, el constituyente destinó ciertos artículos a la formación y desarrollo de la seguridad social en pensiones, concebido como sistema; entre ellos tenemos:

- Artículo 46. En lo que respecta a las personas de la tercera edad, se sostuvo que: el Estado garantizará los servicios de seguridad social.

- Artículo 48. Con su respectiva adición, mediante el art 1 del Acto Legislativo 01 de 2005. Que desarrolla todos los alcances de la seguridad social e introduce el principio de sostenibilidad financiera que nos ocupa.

- Artículo 53. Este es uno de los artículos, que luego de 26 años de expedición de la Carta no se ha materializado, no obstante, mal haríamos en obviarlo, ya que, si bien no ha sido objeto de reglamentación por parte del Congreso evidenciaba el sentir del constituyente. El artículo en mención, conminaba al Congreso a expedir el Estatuto del trabajo, el cual tendría en cuenta por lo menos ciertos principios fundamentales, entre ellos la garantía a la seguridad social, la capacitación, el adiestramiento y el descanso necesario. Del mismo modo, señala la norma que: "El Estado garantiza el derecho al pago oportuno y al reajuste periódico de las pensiones legales".

De este inciso se desprende uno de los factores que conlleva a la reliquidación de las pensiones públicas, como lo es la indexación de la primera mesada pensional, conforme al índice de precios iniciales y finales, establecidos por el DANE.

Artículos transitorios: Es menester señalar que dichos artículos no fueron objeto de regulación de ningún tipo, como tampoco se organizaron o crearon las comisiones que adelante se señalaran.

- Artículo 47 transitorio. La ley organizará para las zonas afectadas por aguda violencia, un plan de seguridad social de emergencia, que cubrirá un periodo de tres años.

- Artículo 57 transitorio. Se creará una comisión integrada por el Gobierno, los sindicatos, los gremios económicos, movimientos 
políticos, campesinos y trabajadores informales, en un plazo de 180 días, para elaborar una propuesta que desarrolle normas de seguridad social.

En este sentido, se evidencia como la seguridad social en el ámbito pensional, adquiere un rango constitucional que soporta y fundamenta su aplicación dentro del ordenamiento jurídico colombiano, sin que ello pueda traducirse en una real y efectiva protección de los derechos que nos ocupan, tal y como se mostrará en el desarrollo de estas líneas.

\section{De los empleados públicos como sujetos de estudio y su régimen pensional: precisiones conceptuales.}

En primer lugar, se hace necesario establecer ciertas delimitaciones del orden conceptual, por ello es menester determinar el campo de acción y el campo de estudio objeto de estas líneas. En lo que respecta al campo de acción, estas observaciones se centran en los empleados públicos como sujetos de Derecho. Ahora bien, estará dirigida al régimen pensional y los requisitos que deben albergar estos sujetos a fin de adquirir el estatus de pensionado.

Nuestros esfuerzos van encaminados al estudio del régimen de prima media con prestación definida y las modificaciones que este ha sufrido en cuanto a sus principios orientadores, es por ello que resulta pertinente hacer ciertas precisiones.

En lo que respecta a las mentadas modificaciones sufridas por el régimen, estas no devienen exclusivamente de la Ley 100 de 1993, "ya que existen múltiples reformas, extraídas a partir de la expedición de la ley 797/2003; las leyes 168 y 712 de 2003; y el acto legislativo 01 de 2005" (Santamaría \& Piraquive, 2013), no obstante, nos centraremos en las reformas introducidas por la ley 100 y el acto legislativo 01 de 2005.

Determinadas las variaciones que ocuparan el devenir de estas líneas, es menester indicar que, en lo atinente al régimen pensional de los empleados públicos, tenemos que, antes de la entrada en vigencia de la ley 100 de 1993 — esto es el 1 de abril de 1994 — se contaba con dos disposiciones normativas que determinaban las condiciones y/o requisitos 
para ser acreedor de la prestación económica que es objeto de la presente investigación, según fuere el caso.

Las disposiciones legales en mención constituían el marco legal del acceso a la pensión hallándose condensado en la Ley 33 de 1985 y la Ley 71 de 1988. De otra parte, con la ley 71 se expidieron normas sobre el régimen pensional del sector público, en particular cuando el empleado público padecía la pérdida de su capacidad laboral o sobrevenía la muerte. Vale decir, que la norma en comento, determinaba las pensiones en ocasión a la pérdida total de la capacidad laboral o por incapacidad permanente parcial; asimismo estaba orientada a hacer explícitos los criterios para acceder a la pensión de sobrevivientes.

Por ello, tenemos que el tema que nos concierne dentro de las presentes reflexiones está orientado a determinar si el objeto del sistema pensional -por vejez-y uno de sus principios orientadores guardan relación o se contraponen, por tanto, resulta pertinente hacer mención de otros regímenes existentes que regulaban esta materia, para indicar que si bien se ha hecho alusión a la Ley 71 de 1988, esta no contara con un desarrollo y quedara en la mera enunciación a fin de entender de forma global los criterios pensionales.

Por otra parte, tenemos el campo de estudio; obedece a criterios conceptuales que se encaminan a la determinación de algunas variables que le permiten a los sujetos de derecho identificar tópicos relacionados con los principios orientadores del sistema general de seguridad social en pensiones y sus fines últimos.

Habiendo delimitado nuestro objeto de estudio, resulta pertinente establecer las relaciones entre Funcionario público, servidor público, empleado público y trabajador oficial. Estas acepciones aparentan ser muy similares a la vista, tanto así, que han venido siendo decantadas por la Sala de Consulta, servicio civil del Consejo de Estado y por la Corte Constitucional. Vemos que la Dirección de Diversidad Sexual de la Secretaría Distrital de Planeación emitió un concepto en la Comunicación de la Secretaría Distrital de Planeación, Radicación 1-2010-55021, 3-201041377, en el que se destacan estas precisiones:

i. Servidor público: La Constitución Política de 1991, en su artículo 123 define los servidores públicos como aquellos miembros de las 
corporaciones públicas, los empleados y trabajadores del Estado y de sus entidades descentralizadas territorialmente y por servicios, quienes están al servicio del Estado y de la comunidad y ejercen sus funciones en la forma prevista por la Constitución, la ley y el reglamento.

La anterior definición fue acogida por la Sala de Consulta y Servicio Civil en su concepto 855 de 19961, al definir al servidor público así:

Servidores públicos es un concepto genérico que emplea la Constitución Política para comprender a los miembros de las corporaciones públicas y a los empleados y trabajadores del Estado y de sus entidades descentralizadas territorialmente y por servicios; todos ellos están «al servicio del Estado y de la comunidad»y deben ejercer sus funciones en la forma prevista en la Constitución, la ley y el reglamento. [Negrillas y subrayado fuera del texto].

ii. Funcionario: Por su parte, la Corte Constitucional en la Sentencia C-681 de 2003, contempló la definición de funcionario público en los siguientes términos:

Las personas naturales que ejercen la función pública establecen una relación laboral con el Estado y son en consecuencia funcionarios públicos... La clasificación tradicional comprende los empleados públicos y los trabajadores oficiales. Esta clasificación se remonta a la ley 4a de 1913 la cual siguiendo el criterio finalista definió a los empleados públicos como los que tienen funciones administrativas y los trabajadores oficiales aquéllos que realizan las obras públicas y actividades industriales y comerciales del Estado. El decreto 3135 de 1968 siguió el criterio organicista para definir los empleados públicos, quienes están vinculados a los Ministerios, departamentos administrativos y demás entidades que ejercen la función pública.

Luego de estas precisiones, podemos abordar el concepto que nos ocupa, pero para ello será necesario diferenciarle de los trabajadores oficiales, quienes aun estando vinculados a entidades públicas gozan de un contrato de trabajo distinto. 
Iniciaremos sosteniendo que el término servidor público comprende a todos los trabajadores que laboran para el Estado. Así pues, los empleados públicos y los trabajadores oficiales son servidores públicos. Dicho en otras palabras, los servidores públicos son el género, y los empleados públicos al igual que los trabajadores oficiales son especies de ese género (Alvarado, 2015).

Son empleados públicos las personas que laboran en los ministerios, en los establecimientos públicos, en las superintendencias, en los departamentos administrativos y sus equivalentes en el ámbito territorial o distrital, en labores distintas de las de construcción y sostenimiento de obras públicas. Los que laboran en esas entidades en la construcción y sostenimiento de obras públicas son trabajadores oficiales.

En este orden de ideas, tenemos que los trabajadores oficiales, quienes laboran en los ministerios, los establecimientos públicos, las superintendencias, los departamentos administrativos y sus equivalentes en el ámbito territorial o distrital, adicional en la construcción y sostenimiento de las obras públicas, así como aquellos que prestan sus servicios en las empresas industriales y comerciales del Estado de cualquier nivel y sin que importe las funciones asignadas al respectivo organismo, con excepción de lo que desempeñen labores de dirección y confianza (Dueñas, 2003).

Por lo antes señalado podemos sostener, que los empleados públicos son una categoría de trabajadores que desempeñan ciertas actividades en las entidades estatales, contando con un contrato de trabajo, que los ubica en del RPMPD dentro del sistema pensional.

\section{Principios orientadores del SGSSP en Colombia, a partir de la Constitución Política de 1991}

Como se sostuvo en su oportunidad, la constitucionalización de las diversas ramas del derecho ha sido un común denominador desde la expedición de la Carta Política de 1991, lo anterior debido en gran parte a las tendencias socialistas que la regentan. Vemos como en el artículo 48 de la constitución (que ya había sido mencionado en el desarrollo temático, sin que fuere objeto de mayores reparos) es el fundamento constitucional que soporta el derecho a la seguridad social en Colombia. Dentro de la norma 
objeto de estudio, el constituyente primario indicó el carácter obligatorio del derecho, afianzando su calificativo de irrenunciable y sosteniendo que la prestación del mismo estaba orientada bajo los principios de eficiencia, universalidad y solidaridad.

Para el desarrollo de estos principios podemos acudir a la jurisprudencia constitucional; en Sentencia C-258 de 2013, con el Magistrado ponente Jorge Ignacio Pretelt Chaljub la Corte Constitucional hizo un análisis cuidadoso de la composición y alcance de esta garantía constitucional. En una primera medida, explicó el principio de eficiencia y la definición que la Corte le ha endilgado a este principio:

Por su parte, el principio de eficiencia requiere la mejor utilización social y económica de los recursos humanos, administrativos, técnicos y financieros disponibles, para que los beneficios a que da derecho la seguridad social, sean prestados en forma adecuada, oportuna y suficiente. La jurisprudencia de esta Corporación ha definido la eficiencia como la elección de los medios más adecuados para el cumplimiento de los objetivos y la maximización del bienestar de las personas [Subrayado fuera del texto].

Del mismo modo, la corporación en mención en la jurisprudencia que nos ocupa agotó el principio de universalidad, señalando al estado como sujeto pasivo principal de este derecho, y por tanto, manifestando la obligación expresa de garantizar dichas prestaciones:

... Según el principio de universalidad, el Estado -como sujeto pasivo principal del derecho a la seguridad social-debe garantizar las prestaciones de la seguridad social a todas las personas, sin ninguna discriminación, y en todas las etapas de la vida. Por tanto, el principio de universalidad se encuentra ligado al mandato de ampliación progresiva de la cobertura de la seguridad social señalado en el inciso tercero del mismo artículo 48 constitucional, el cual a su vez se refiere tanto a la ampliación de afiliación a los subsistemas de la seguridad social -con énfasis en los grupos más vulnerables-, como a la extensión del tipo de riesgos cubiertos. 
En consecuencia, se hace necesario definir el principio de solidaridad, para ello acudimos a la sentencia T-045 de 2016, con el Magistrado ponente Jorge Ignacio Pretelt Chaljub, en la cual se aborda desde las visiones de la mutua ayuda, propias del artículo 95 de la constitución que, dentro de los deberes y obligaciones del ciudadano, señala que debe actuarse conforme al principio de solidaridad social, así las cosas, se indica que:

... la solidaridad, hace referencia a la práctica de la mutua ayuda entre las personas, las generaciones, los sectores económicos, las regiones y las comunidades. Este principio tiene dos dimensiones: de un lado, como bien lo expresa el artículo 2 de la Ley 100 de 1993, hace referencia a que el Estado tiene la obligación de garantizar que los recursos de la seguridad social se dirijan con prelación hacia los grupos de población más pobres y vulnerables; de otro, exige que cada cual contribuya a la financiación del sistema de conformidad con sus capacidades económicas, de modo que quienes más tienen deben hacer un esfuerzo mayor [Subrayado fuera del texto].

Adicional a estos principios, se estableció que el Estado con la participación de los particulares, ampliara la cobertura de la seguridad social, a lo anterior, no podemos darle el alcance de principio orientador ni de objeto del sistema, ya que hasta este punto no se había establecido expresamente.

- En este sentido, los principios constitucionales mencionados orientan hasta nuestros días el sistema pensional, sin embargo, a raíz de las reformas introducidas al sistema se sumaron otros. En este orden de ideas, la Ley 100 de 1993 cuya entrada en vigencia fue el 1 de abril de 1994, afianzó los principios de la carta, esto es: eficiencia, universalidad y solidaridad; al mismo tiempo integro otros, entre ellos: integralidad, unidad y participación; no obstante, en su artículo decimo manifestó expresamente que el objeto del sistema era propender hacia la ampliación progresiva de cobertura a los segmentos de población no cubiertos con un sistema de pensiones. En este momento, si es viable aseverar que el sistema contaba con un objeto, entendido este último como el fin último del quehacer del sistema. 
Estos principios orientadores incluidos por la ley 100, en su artículo 2 se definieron, señalando que la integralidad es la cobertura de todas las contingencias que afectan la salud, la capacidad económica y en general las condiciones de vida de la población. Para este efecto, cada persona contribuye según su capacidad y recibe lo necesario para atender sus contingencias. Por su parte, la unidad es la articulación de políticas, instituciones, regímenes, procedimientos, así como prestaciones para alcanzar los fines de la seguridad social; mientras que la participación es la intervención de la comunidad a través de los beneficios de la seguridad social en la organización, control, gestión y fiscalización de las instituciones y del sistema en su conjunto. Estos conceptos, sumados a la progresividad, aportan una mejor comprensión de los alcances que de ellos fija la Carta.

Por ello, no fue necesario acudir a un desarrollo vía jurisprudencia para establecer significados o determinar alcances. En este sentido, es menester indicar que la Corte Constitucional, en reiterada jurisprudencia ha utilizado estos conceptos sin que diera lugar a nuevas apreciaciones en el orden jurisprudencial, esto se observa en la Sentencia C408 de 1994 con Magistrado Ponente Dr. Fabio Morón Díaz, del mismo modo en la Sentencia C-760 de 2004, con el Magistrado ponente Dr. Rodrigo Uprimny Yepes.

Por otro lado, tenemos la consolidación del objeto del sistema de manera expresa, pero esto será tratado en el siguiente aparte; por lo pronto, nos concentraremos en las modificaciones introducidas por el Acto Legislativo 01 de 2005. Este acto reformatorio de nuestra carta introdujo el principio de sostenibilidad financiera al sistema de seguridad social en pensiones, además planteó la necesidad de propender a la protección de los derechos adquiridos.

En lo que ha derechos adquiridos se refiere, estos han sido objeto de múltiples pronunciamientos jurisprudenciales. Lo anterior, conforme a la definición de los alcances del principio de progresividad y no regresividad que regenta a la seguridad social; debido a que la Corte ha establecido la distinción entre los derechos adquiridos y las meras expectativas (esta última obedece al derecho civil).

En este sentido, la Corte ha sostenido en sentencia T-045 de 2016 que "configuran derechos adquiridos las situaciones jurídicas individuales 
que han quedado definidas y consolidadas bajo el imperio de una ley y que, en tal virtud, se entienden incorporadas válida y definitivamente o pertenecen al patrimonio de una persona"; es decir, que para configurar un derecho adquirido, es necesario que se reúnan todas las condiciones necesarias para adquirirlo antes de que opere el tránsito legislativo. De tal manera, las meras expectativas "son aquellas esperanzas o probabilidades que tiene una persona de adquirir en el futuro un derecho, si no se produce un cambio relevante en el ordenamiento jurídico".

En este orden de ideas, podríamos aseverar que una de las diferencias entre estas dos instituciones radica en que, mientras los derechos adquiridos gozan de la garantía de firmeza e inmutabilidad que se deriva de su protección expresa en la Constitución (art 58), salvo casos excepcionales, las meras expectativas, por el contrario, pueden ser objeto de modificación por el legislador, pues carecen de dicha protección constitucional.

Ahora bien, en lo atinente a las expectativas legítimas y derechos adquiridos en materia pensional, la Corte Constitucional a partir de la sentencia C-789 de 2002, ha sostenido que:

... Si bien es cierto, tratándose de meras expectativas no aplica la prohibición de regresividad, ello no significa que estén desprovistas de toda protección, pues cualquier transito normativo no solo debe consultar los principios de razonabilidad y proporcionalidad, sino que, además, en función del principio de confianza legítima, se debe proteger la creencia cierta del administrado de que la regulación que lo ampara en un derecho se seguirá manteniendo vigente en el ordenamiento jurídico... cuánto más cerca está una persona de acceder al goce efectivo de un derecho, mayor es la legitimidad de su expectativa en este sentido.

En este contexto, luego de la sentencia C-789 de 2002, la Corte ha indicado:

En la jurisprudencia constitucional ha surgido una categoría intermedia entre derechos adquiridos y meras expectativas, denominada "expectativas legítimas", concepto que hace referencia a que en determinados casos se puede aplicar el principio de no regresividad a las aspiraciones pensionales 
próximas a realizarse de los trabajadores, cuando se trata de un cambio de legislación abrupto, arbitrario e inopinado, que conduce a la vulneración del derecho al trabajo de manera desproporcionada e irrazonable [Subrayado fuera del texto].

De lo anterior se colige que en lo que respecta al principio de progresividad y no progresividad, en Colombia podemos encontrarnos en presencia de los derechos adquiridos, las expectativas legítimas y las meras expectativas. En lo que atañe al principio de sostenibilidad financiera, lo abordaremos en contraste con el objeto del sistema.

\section{El objeto del SGSSP a la luz del principio de Sostenibilidad Financiera}

Como se ha dicho, el alcance de este estudio pretende establecer ciertos tópicos que permitan soportar que el objeto del sistema y el principio de sostenibilidad financiera no responden a intereses similares, lo cual puede traducirse en que un principio orientador del sistema que en cierta medida podría estar en contraposición al fin último del sistema que orienta.

Abordando estas dimensiones, es menester iniciar con una serie de distinciones, por tanto, se hace necesario aclarar qué debe entenderse por cobertura. La cobertura no se asemeja a la posibilidad de cotizar o no a pensiones —esta preocupación es del orden económico-. La cobertura obedece a ser acreedor de la prestación económica denominada pensión, es decir, lograr el reconocimiento de dicha prestación (Duque \& Duque, 2016).

En este sentido, se hace necesario traer a colación un pronunciamiento realizado por la Corte Constitucional, que en sentencia C-078 de 2017, con el Magistrado Ponente Jorge Iván Palacio Palacio, en la cual se enfatizó que la mentada corporación ya se había pronunciado sobre la cobertura en reiteradas oportunidades, tales como en las sentencias C-258 de 2013, C-623 y 1024 de 2004, cuando se desarrolló el principio de universalidad, desglosando a la cobertura como una extensión del mentado principio, sostuvo que:

El principio de universalidad referido a la cobertura en la protección de los riesgos inherentes a la seguridad social 
debe amparar a todas las personas residentes en Colombia, en cualquiera de las etapas de su vida, sin discriminación alguna por razones de sexo, edad, raza, origen nacional o familiar, lengua, religión, opinión política o filosófica, etc. [Subrayado fuera del texto].

De otra parte, la sostenibilidad financiera procura por el mantenimiento y pervivencia del sistema. Esto es un criterio del orden económico-administrativo, que propende al sostenimiento del sistema, con la inmersión de nuevos cotizantes, a partir del relevo generacional. "Ampliar la cobertura representa el reconocimiento de prestaciones, mientras que sostener financieramente el sistema responde a medidas de protección económicas" (Corte Constitucional, sentencia C-111 de 2006).

En este orden de ideas, la operacionalización del sistema indica que, las mesadas pensionales son productos de los aportes realizados por los acreedores (mal llamados beneficiarios de la prestación económica), pero no totalmente, sino que dependen de los aportes que hacen quienes no están pensionados (algo así como una pirámide), y si los aportes de los nuevos no alcanzan para financiar los pagos a los acreedores de la prestación, el Estado debe financiar esos pagos, lo cual hace vía impuestos, razón por la que este régimen está condenado a la desaparición, porque matemáticamente (aun cuando se propugna por su sostenibilidad financiera) no es autosostenible.

Por ello, en el ámbito nacional vemos como se niegan pensiones por requisitos mínimos, que nos hacen creer en la existencia de una política pública, que hemos denominado "la carrera de la negación". Esto, aunque suene descabellado, es una medida - perversa- que garantiza la sostenibilidad del sistema.

\section{Referencias}

Alvarado, L. (2015). La Unificación y Extensión de la Jurisprudencia del Consejo de Estado en el Régimen de Transición Pensional en Colombia. (Tesis de Maestría). Universidad Militar de Colombia.

Dueñas, O. (2003). Las Pensiones. Teoría, normas y Jurisprudencia. 2a ED. Bogotá: Ediciones del Profesional.

Duque, S., \& Duque, N. (2016). El derecho fundamental a una pensión y el principio de sostenibilidad financiera: un análisis desde el régimen 
de prima media con Prestación definida en Colombia. Justicia Juris, 12(1), 40-55.

Guerrero, G. (2008). El derecho laboral en la constitución nacional. Cartagena: Fundación Tecnológica Antonio de Arévalo TECNAR.

Corte Constitucional (2002). Sentencia C-789. Exp. D-3958. [M. P. Rodrigo Escobar Gil].

Corte Constitucional (2002). Sentencia 1027. Exp. D-4027. [M.P. Clara Inés Vargas Hernández].

Corte Constitucional (2006). Sentencia C-862. [M. P. Humberto Antonio Sierra Porto].

Corte Constitucional (2006). Sentencia C-111. Exp. D-5899 [M. P. Rodrigo Escobar Gil].

Corte Constitucional (2006). Sentencia C-792. Exp. D-6242. [M. P. Rodrigo Escobar Gil].

Corte Constitucional, Sala Cuarta de Revisión (6 de septiembre de 2007). Sentencia T-696 Exp. T-1616436 [M. P. Rodrigo Escobar Gil].

Corte Constitucional (2007). Sentencia T-045. Exp. T-1429693. [M. P. Jaime Córdoba Triviño].

Corte Constitucional (2007). Sentencia T-224 [M. P. Rodrigo Escobar Gil].

Corte Constitucional (2011). Sentencia T-762. Expediente T-3085282. [M. P. María Victoria Calle Correa].

Corte Constitucional (2013). Sentencia T-640. Exp. T-3085282. [M. P. Mauricio González Cuervo].

Corte Constitucional (2013). Sentencia C-258. Exp. D-9173 y D-9183. [M. P. Jorge Ignacio Pretelt Chaljub].

Corte Constitucional (2016). Sentencia T-045. Exp. T-5189723. [M. P. Jorge Ignacio Pretelt Chaljub].

Congreso de Colombia (24 de junio de 1948). Sobre Procedimientos en los juicios del Trabajo. Código Procesal del Trabajo adoptado por el Decreto 4133 de 1948 como legislación permanente [Ley 2158 de 1948].

Congreso de Colombia (29 de enero de 1985). Por la cual se dictan algunas medidas en relación con las Cajas de Previsión y con las prestaciones sociales para el Sector Público [Ley 33 de 1985]. 
Congreso de Colombia (19 de diciembre 1988). Por la cual se expiden normas sobre pensiones y se dictan otras disposiciones. Reglamentado por el Decreto Nacional 1073 de 2002 [Ley 71 de 1988].

Congreso de Colombia (23 de diciembre 1993). Ley 100 de. Por la cual se crea el sistema de seguridad social integral y se dictan otras disposiciones [Ley 100 de 1993].

Congreso de Colombia (29 de enero 2003). Por la cual se reforman algunas disposiciones del sistema general de pensiones previsto en la Ley 100 de 1993 y se adoptan disposiciones sobre los Regímenes Pensionales exceptuados y especiales [Ley 797 de 2003].

Congreso de Colombia ( 5 de diciembre de 2003). Por el cual se reforma el Código Procesal del Trabajo [Ley 712 de 2003].

Santamaría, M. \& Piraquive, G. (2013) Evolución y alternativas del sistema pensional en Colombia. Archivos de Economía. Documento 398. Bogotá: Departamento Nacional de Planeación. 